\title{
Editorial
}

\section{Propagation Phenomena and Transitions in Complex Systems: Efficient Mathematical Models}

\author{
Cristian Toma, ${ }^{1}$ Ezzat G. Bakhoum, ${ }^{2}$ and $\mathrm{Ming}^{\mathrm{Li}}{ }^{3}$ \\ ${ }^{1}$ Department of Applied Sciences, Politehnica University, 060042 Bucharest, Romania \\ ${ }^{2}$ University of West Florida, Pensacola, FL 32514, USA \\ ${ }^{3}$ Department of Electronic Science and Technology, School of Information Science and Technology, \\ East China Normal University, Shanghai 200062, China
}

Correspondence should be addressed to Cristian Toma, cgtoma@physics.pub.ro

Received 14 September 2011; Accepted 14 September 2011

Copyright (c) 2012 Cristian Toma et al. This is an open access article distributed under the Creative Commons Attribution License, which permits unrestricted use, distribution, and reproduction in any medium, provided the original work is properly cited.

Today, engineers face an increasing challenge in advanced applications that are based on efficient mathematical models for propagation and transition phenomena. Propagation aspects implying commutative and/or additive consequences of quantum physics are used extensively in the design of long-range transmission systems. Differential geometry is adapted for solving nonlinear partial differential equations with very great number of variables for transitions in complex optoelectronics systems. Special mathematical functions are used in modeling very small-scale material properties (energy levels and induced transitions) in quantum physics for the design of nanostructures in microelectronics. Time series with extremely high transmission rates are used for multiplexed transmission systems for large communities. All these advanced engineering subjects require efficient mathematical models in the development of classical tools for complex systems such as differential geometry, vector algebra, partial differential equations, and time-series dynamics. The objective in such applications is to take into consideration efficiency aspects of mathematical and physical models required by basic phenomena of propagation and transitions in complex systems, particularly in situations implying physical limits as long-distances propagation phenomena, quantum transitions in nanostructures, complex systems with great number of variables, and infinite spatiotemporal extension of material media.

The aim of this special issue is to present high qualitative and innovative developments for efficient mathematical approaches of propagation phenomena and transitions in complex systems with applications in physics and engineering. Using efficient mathematical tools for modeling propagation and transition phenomena, for solving nonlinear partial 
differential equations describing wave propagation, and for analyzing the transitions of complex systems, significant results were obtained in the research fields of low-scale physical phenomena, heterogenous structures for microelectronics, dynamics of complex systems (including networks and industrial systems), and efficient signal and image analysis based on fundamental mathematical and physical laws.

This special issue involves 27 original papers, selected by the editors so as to present the most significant results in the previously mentioned topics. These papers are organised as follows.

(a) There are nine papers on advanced mathematical tools for analyzing propagation, fluctuations, long-distance similarities, singularities, and dynamics in complex systems: "Complexity on acute myeloid leukemia mRNA transcript variant" by C. Cattani and G. Pierro, "A class of negatively fractal dimensional Gaussian random functions" by M. Li, "Specific mathematical aspects of dynamics generated by coherence functions" by E. G. Bakhoum and C. Toma, "Viewing sea level by a one-dimensional random function with long memory" by M. Li et al. "Exact solution of impulse response to a class of fractional oscillators and its stability" by M. Li et al. "Mixture of generalized gamma density-based score function for fastica" by M. El-Sayed Waheed et al., "A sextuple product identity with applications" by J. M. Zhu, "Robust L2-L攼-infinite filtering of time-delay jump systems with respect to the finite-time interval" by S. He and F. Liu, and "Enhanced cryptography by multiple chaotic dynamics" by J. Chen et al.

(b) There are ten papers on efficient mathematical models for propagation and transition phenomena for specific applications: "Recent advancements in fractal geometricbased nonlinear time series solutions to the micro-quasistatic thermoviscoelastic creep for rough surfaces in contact" by O. M. Abuzeid et al., "Long memory from Sauerbrey equation: a case in coated quartz crystal microbalance in terms of ammonia" by X.-H. Wang et al., "Approximate method for studying the waves propagating along the interface between air-water" by M. M. Khader, and R. F. Al-Bar, "Rayleigh wave in generalized magnetothermo-viscoelastic granular medium under the influence of rotation, gravity field, and initial stress" by A. M. Abd-Alla et al., "An application of Saint Venant's theory to Volterra's distortions" by I. Bochicchio et al., "Generalized Jacobi elliptic function solution to a class of nonlinear Schrödinger-type equations" by Z. Al-Mhuaimeed and E. Abdel-Salam, "Trigonometric function periodic wave solutions and their limit forms for the KdV like and the PC like equations" by L. Zhengrong et al., "Exact solution for the time-dependent temperature field in dry grinding: application to segmental wheels" by J. L. GonzlezSantander et al., "The inverse fundamental operator marching method for Cauchy problems in range-dependent stratified waveguides" by $\mathrm{P}$. Li and W. Zhong, and "Investigation of patch antenna based on photonic band-gap substrate with heterostructures" by Z. Li et al.

(c) There are eight papers on transition and propagation in artificial complex systems (networks, image analysis, and industrial systems): "Modeling and analysis of re-entrant manufacturing systems: micro- and macroperspectives" by F. He et al., "Selftuning random early detection algorithm to improve performance of network transmission" by J. Chen et al., "Mbm-based scalings of traffic propagated in internet" by M. Li et al., "Mixed variables-attributes test plans for single and double acceptance sampling under exponential distribution" by Y. Li et al., "Enclosed Laplacian operator of nonlinear anisotropic diffusion to preserve singularities and delete isolated points in image smoothing" by Z. Liao et al., "A new numerical method for preserving curve edges in nonlinear 
anisotropic smoothing" by S. Hu et al., "Robust affine invariant descriptors" by J. Yang et al., and "Improved generalized belief propagation for vision processing" by S. Chen et al.

Cristian Toma

Ezzat G. Bakhoum

Ming Li

\section{Acknowledgments}

M. Li would like to acknowledge the support by the 973 plan under the project no. 2011CB302802, and by the NSFC under the project Grant nos. 61070214, 60873264. 


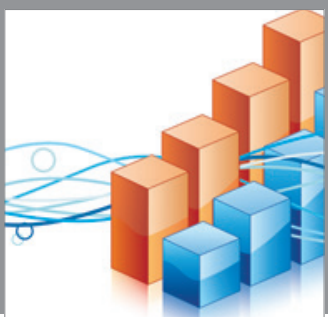

Advances in

Operations Research

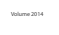

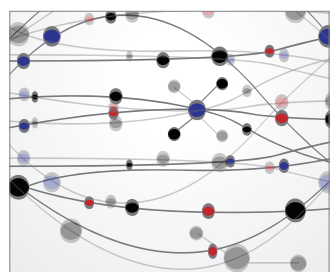

\section{The Scientific} World Journal
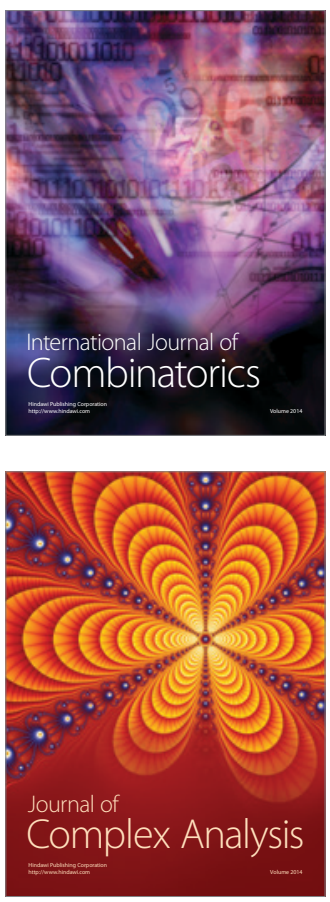

International Journal of

Mathematics and

Mathematical

Sciences
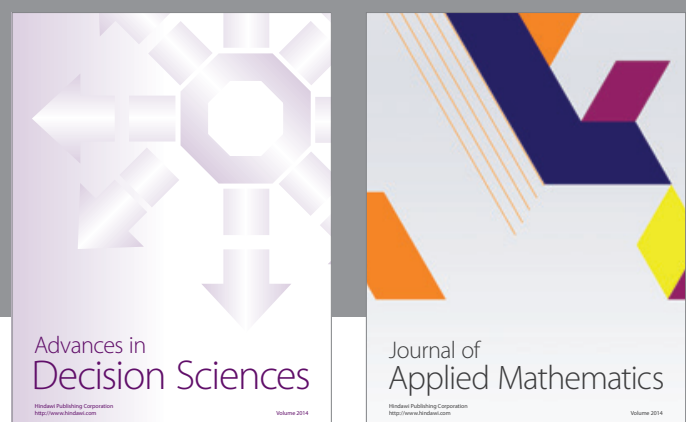

Journal of

Applied Mathematics
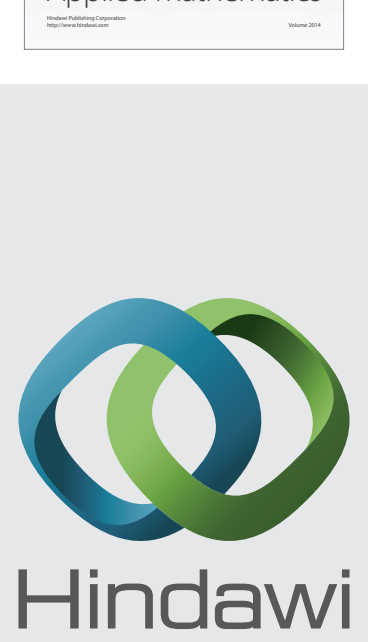

Submit your manuscripts at http://www.hindawi.com
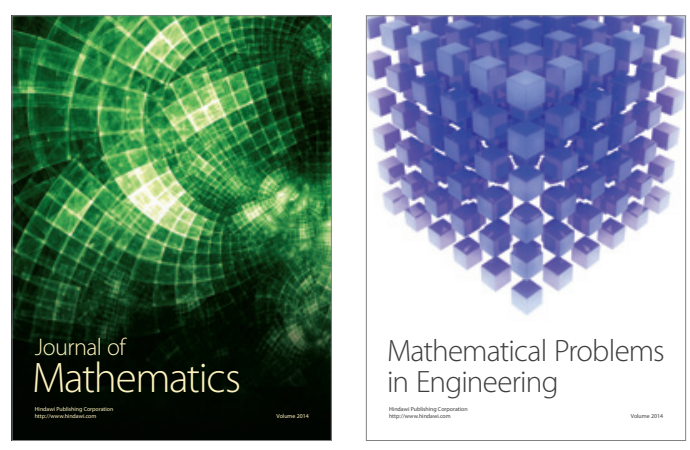

Mathematical Problems in Engineering
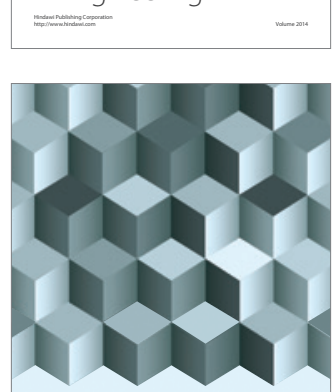

Journal of

Function Spaces
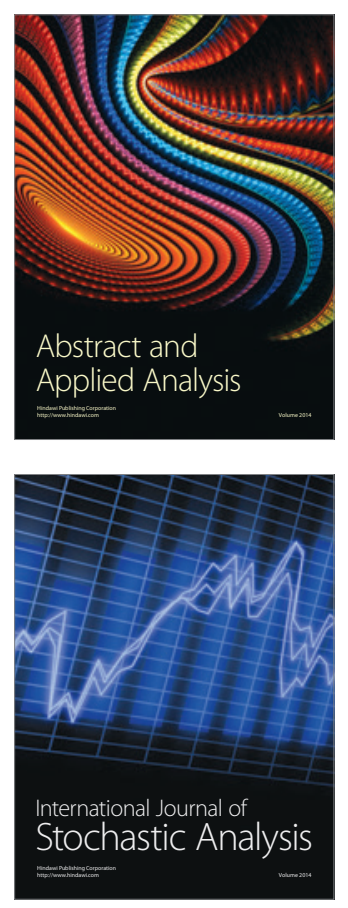

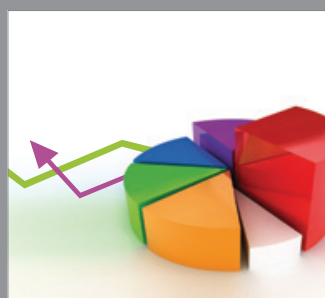

ournal of

Probability and Statistics

Promensencen
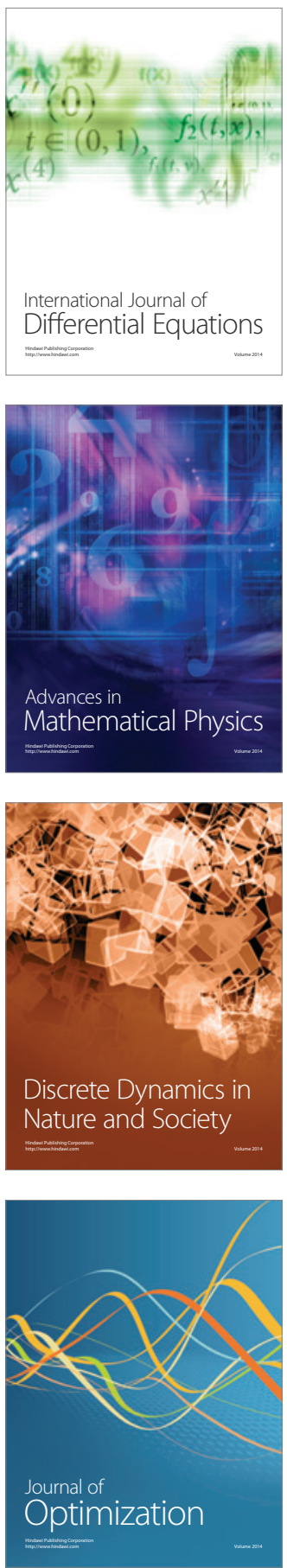